\title{
Análisis de las impresiones diagnósticas en consultantes de un Centro de Atención Psicológica Universitario ${ }^{6}$
}

Mariela Narváez Marín

$\mathrm{PhD}(\mathrm{C})$. Salud Pública Especialista en Farmacodependencia Universidad de Manizales Manizales, Colombia Correo electrónico: maye888@gmail.com

\section{Héctor Haney Aguirre-Loaiza \\ $\mathrm{Ms}(\mathrm{C})$ en Psicología. Psicólogo Universidad del Valle Cali, Colombia}

\section{Resumen}

Objetivo: describir y comparar las impresiones diagnósticas entre varones y mujeres, así como identificar la evolución de consultas entre los años 2006 y 2011, atendidas en el Centro de Atención Psicológica de la Universidad de Manizales. Método: corresponde a un estudio con metodología cuantitativa, ex post facto. Se analizaron 1505 historias clínicas, de las cuales el 48.9\% ( $n=736)$ correspondió a las mujeres. Resultados: se muestran problemas adaptativos, conyugales, depresivos y paterno-filiales, como los más prevalentes para ambos sexos. Las mujeres presentan más los problemas adaptativos en comparación con los varones, mientras que en estos son mayores los problemas conyugales, depresivos y paterno-filiales. Se realizan discusiones a la luz de informes de salud mental y trabajos relacionados con poblaciones escolarizadas.

Palabras clave

Salud Mental, Diagnóstico, Trastornos Mentales, Historia del paciente. (PsyclNFO - Thesaurus).

6 Para citar este artículo: Narváez, M., \& Aguirre-Loaiza, H. (2016). Análisis de las impresiones diagnósticas en consultantes de un Centro de Atención Psicológica Universitario. Informes Psicológicos, 16(2), pp. 87-101. http:// dx.doi.org/10.18566/infpsicv16n2a06 


\section{Analysis of the diagnostic impressions in a University counseling center consultants}

\section{Abstract}

Objective: to describe and compare the diagnostic impressions between men and women, and to identify the evolution of consultation between 2006 and 2011, met in the Centre of Psychological Care at the University of Manizales. Method: an ex post facto, quantitative study was done. 1505 medical records were analyzed of which $48.9 \%(n=736)$ corresponded to women. Results: the most prevalent problems for both sexes are adaptive, depressive and parent-child, as well as marital. Women have more adaptive problems in comparison with men, whereas men have more depressive, marital and parent-child related issues. Discussions take place in the light of reports of mental health and work related to uneducated populations.

\section{Análise das impressões diagnósticas em consultantes de um centro de atenção psicológica universitário}

\section{Resumo}

Objetivo: descrever e comparar as impressões diagnósticas entre homens e mulheres, assim como identificar a evolução de consultas entre os anos 2006 e 2011, atendidas no Centro de Atenção Psicológica da Universidade de Manizales. Método: corresponde a um estudo com metodologia quantitativa, ex post facto. Se analisaram 1505 histórias clínicas, das quais 48.9\% $(n=736)$ correspondeu às mulheres. Resultados: se mostram problemas adaptativos, conjugais, depressivos e paterno-filiais, como os mais prevalentes para ambos sexos. As mulheres apresentam mais problemas adaptativos em comparação com os homens, enquanto que nestes são maiores os problemas conjugais, depressivos e paterno-filiais. Se realizam discussões à luz de informes de saúde mental e trabalhos relacionados com populações escolarizadas.

Palavras chave saúde mental, diagnóstico, transtornos mentais, história do paciente. (PsycINFO - Thesaurus). 


\section{ntroducción}

Colombia cuenta con 106 Programas activos de Psicología ofrecidos por 85 instituciones de educación superior, con características de heterogeneidad en cuanto a créditos, costo de las matrículas y condiciones de calidad, mientras que, por otro lado, presentan un estatus de homogeneidad en aspectos relacionados con la metodología, la jornada y la duración (Montoya, 2011). En tal panorama, el contexto educativo en la formación de psicólogos en Colombia ha presentado drásticos cambios y dinámicas fluctuantes que, como consecuencia, generan ciertas rupturas y problemáticas (Organista \& Garzón, 2012). Bajo esta lógica, la perspectiva de varios Programas de Psicología, unfica sus esfuerzos para tratar de resignificar el proceso educativo, centrados en la estructura del binomio enseñanza-aprendizaje, y los cuales se ciñen principalmente a las prácticas pedagógicas.

Es así como Organista y Garzón (2012) consideran que una de tales problemáticas, y a su vez un reto para los programas de psicología, se enmarca tanto en su enseñanza como en su práctica, la cual debe hacerse dentro y fuera de los salones de clase, y con medios e información que satisfagan los requerimientos del contexto sociocultural de cada programa.

Lo anterior no tiene sentido sin la relación directa y coherente entre la naturaleza de la práctica psicológica como disciplina científica, unida a los procesos de investigación, gestión y proyección social. En este contexto, los escenarios de actuación psicológica divergen de acuerdo con el área de conocimiento (clínica, social, educativa, de las organizaciones). No obstante, un foco de aplicación disciplinar que actualmente ocupa el desempeño profesional de los psicólogos colombianos corresponde a la psicología clínica (Sierra \& Fonseca, 2012). En consecuencia, configurar la fuerza que ejerce la articulación del binomio enseñanzaaprendizaje, investigación y proyección social, es reconocido en el territorio colombiano como Centros de Atención Psicológica (CAP) o con otros nombres, como Unidades de Servicio Mental (USP), pero en esencia cumplen las mismas funciones.

La estrategia de los CAP y USP constituye un modelo metodológico asumido por instituciones de educación superior, con distintas aproximaciones e intervenciones, y con intereses particulares de acuerdo con la población a la cual ofrecen los respectivos servicios. Ahora bien, la población es heterogénea; el común denominador de los CAP se centra en el fortalecimiento de la formación de psicólogos (Viñas \& Hernández, 2012). Tal es el caso de la Universidad de Manizales que ofrece el servicio a la población en general, orientado al fortalecimiento de los psicólogos en formación, especialmente en su último año, quienes realizan allí su práctica.

Algunas universidades, en diferentes países, han documentado y destacado procesos de investigación en concordancia con las actividades realizadas en los CAP. Por ejemplo, en España se hizo la descripción de los servicios psicológicos en 74 universidades españolas (Saúl, López, \& Bermejo, 2009); sin embargo, previamente se habían reportado otros 
estudios que señalaban diseños de intervención en la orientación psicológica (Bausela, 2005). Esta tendencia se observa también, desde años anteriores, en otros países europeos y norteamericanos (Bisquerra, 1998) y que aún tienen fuerza en los estudios recientes sobre servicios prestados en los Centros de Atención Psicológica (Castonguay, Locke, \& Hayes, 2011; Hayes, Locke, \& Castonguay, 2011). Por el lado del contexto latinoamericano, algunas de las tendencias se describen con investigaciones que hacen aproximaciones a los desarrollos de los CAP como mecanismos de formación de psicólogos, como también trabajos descriptivos en torno a dichos centros. Entre estos países están: Chile (Loubat \& Magaña, 2005), Argentina (Quesada, 2004), Ecuador (Calvopiña \& Rojeab, 2011) y México (Viñas \& Hernández, 2012).

Entre tanto, en Colombia se encuentran recientes estudios que permiten evidenciar características sociodemográficas de la población, motivos de consulta e impresiones diagnósticas (Ballesteros, Medina, \& Caycedo, 2006; Moreno, Rozo, Cantor, \& Toro, 2012; Muñoz-Martínez \& Novoa-Gómez, 2012; Quesada, 2004). Aunque son varias las universidades colombianas con programas de CAP y USP, son muy escasas aquellas que hacen el ejercicio de divulgación de los respectivos procesos. En consecuencia, se desconoce el comportamiento de los usuarios que asisten a estos centros de atención en otras regiones del país. Pues como queda ratificado a partir de las referencias rastreadas, la visibilización de los resultados se centra en algunas universidades de la ciudad de Bogotá. Aunque cabe mencionar el estudio realizado por Londoño et al. (2010), donde participaron 490 usuarios de varias universidades de Colombia, distribuidos por varias regiones del país; no obstante, la participación de usuarios es pequeña para la cobertura que tienen estos Centros y Unidades de Servicio.

Visto desde otro orden, es necesario resaltar la importancia que revierte este tipo de procedimientos, ya que en esencia permiten destacar el impacto y la relevancia en la práctica clínica del psicólogo en formación, y abriga el papel mediador de la universidad ante problemáticas sociales, y más aún cuando facilitan aportes considerables sobre el estado actual y arrojan resultados del panorama de la salud mental de sus usuario, para futuras tomas de decisiones y puestas en escena de programas de intervención focalizados a la salud mental.

Agregado a esto, el gasto público en salud mental en países en vía de desarrollo está muy por debajo de lo requerido, puesto que el porcentaje del presupuesto asignado a salud mental es mucho menor en estos países (Saxena, Thornicroft, Knapp, \& Whiteford, 2007). Por ejemplo, según datos del Proyecto Atlas (2005) de la Organización Mundial de la Salud (OMS), Colombia presenta la cifra más baja (.1) del porcentaje del presupuesto sanitario total, al lado de Paraguay y por debajo de Bolivia, lo mismo que el número de profesionales dedicados a la salud mental, lo que confirma la insuficiencia de recursos destinados a este sector (Kohn et al., 2005). Las estadísticas en esta materia, han señalado que en Colombia, según el Estudio Nacional de Salud Mental realizado con una muestra de 2.100 participantes (Torres \& Montoya, 1997), para la depresión se reportaron cifras del 5 al 9\% 
para la población femenina y del 2 al 3\% para la población masculina. En un estudio posterior realizado en 2003 , se reportó un incremento con respecto al estudio anterior, donde el 14.9\% de la población femenina y el $8.6 \%$ de la masculina habían presentado un episodio depresivo a lo largo de la vida (Posada et al., 2004).

En consecuencia, se puede afirmar, con plena contundencia, que programas de promoción y prevención, al igual que estrategias de intervención en materia de salud mental, en grupos poblacionales que consultan estos servicios sean estrategias operativas que contribuyen al mejoramiento de la calidad de vida de estas personas. Todo esto cobra sentido si se logran emplear estudios con características epidemiológicas en entidades y programas de atención psicológica ofrecidos por las universidades, al igual que en instancias gubernamentales. En coherencia con lo planteado, los objetivos propuestos para este estudio fueron: (a) identificar la evolución del número de consultas a través de los años analizados, (b) describir y comparar las impresiones diagnósticas de las historias clínicas, entre varones y mujeres.
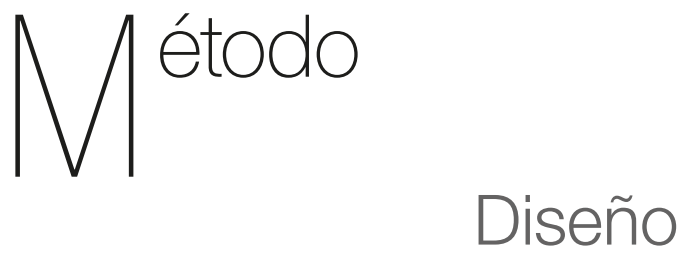

Siguiendo a Montero y León (2005, 2007), el presente estudio pertenece al grupo de planes empíricos con metodología cuantitativa, caracterizados por la tradición objetivista, ex-post facto, al igual que vincula la revisión documental que, para el presente estudio, se tratan de las históricas clínicas.

\section{Unidad de Análisis}

Se revisó un total de 1505 historias clínicas diligenciadas durante un periodo comprendido entre el 2006 y el 2011. Para la evolución de las historias clínicas se tiene un formato general el cual es diligenciado por cada estudiante que realiza su práctica clínica, bajo la supervisión del docente asesor. Todos los documentos reposan en el Centro de Atención Psicológica de la Universidad de Manizales.

Cada caso tuvo el manejo y la responsabilidad pertinente en el registro de la información, siendo acorde a las disposiciones de la ley 1090 del 2006 (Ley del Psicólogo) y las demás consideraciones contempladas con fines de investigación científica expuestas en los apartados del Código de Bioética y Deontológico, regulado por el Colegio Colombiano de Psicólogos (2012).

Procedimiento y análisis de los datos

A cada historia clínica se le asignó un número de identificación, proporcionando datos que fueron procesados y codificados en la matriz de datos. En las columnas se asignaron las variables (sociodemográficas y variables de interés clínico) en las filas las respectivas historias clínicas. Las historias clínicas fueron procesadas en una matriz de datos a través del programa de Microsoft Excel, mediante el cual se establecieron las respectivas codificaciones. El análisis estadístico se hizo mediante el programa SPSS, permitiendo relacionar las variables a través de tablas de contingencia y datos descriptivos de frecuencias y porcentajes. 


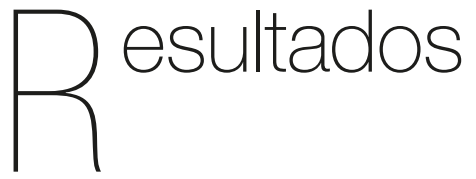

Del total de las historias clínicas analizadas, el 48.9\% ( $n=736)$ pertenecía a las mujeres. En cuanto a la edad, la Tabla 1 describe la distribución en grupos etarios. La gran mayoría son personas menores de 30 años, las cuales representan el 82.5\% de la población atendida. Los jóvenes adolescentes presentaron el mayor porcentaje (34.1\%) con edades correspondientes entre los 16 y 22 años, seguidos de los que tienen entre 9 y 15 años (17.8\%) y, en tercer lugar, las personas con edades comprendidas entre los 23 y los 29 años (16.6\%).

Tabla 1.

Distribución de la población según grupos de edad

\begin{tabular}{cccc}
\hline $\begin{array}{c}\text { Grupo de } \\
\text { edad }\end{array}$ & $\mathbf{n}(\%)$ & Grupo de Edad & n (\%) \\
\hline 2 a 8 años & $211(14.0)$ & 37 a 43 años & $78(5.2)$ \\
9 a 15 años & $268(17.8)$ & 44 a 50 años & $44(2.9)$ \\
16 a 22 años & $513(34.1)$ & 51 a 57 años & $23(1.5)$ \\
23 a 29 años & $250(16.6)$ & 58 a 64 años & $3(.2)$ \\
30 a 36 años & $113(7.5)$ & Más de 65 años & $2(.1)$ \\
\hline
\end{tabular}

La relación de la asistencia al CAP por parte de usuarios, permite establecer la comparación entre la primera y segunda parte de los años analizados (Tabla 2 y Figura 1) siendo el segundo semestre del año donde mayor número de consultas se reportan. Esta tendencia es dominante a excepción del año 2008 donde es superior la cantidad de consultas solicitadas en el primer semestre. Así mismo, se registra que el total de cada año van en disminución en servicios atendidos desde el año 2006 al 2010, en tanto el 2011 (16.2\%) tiene un leve ascenso similar al reportado en el 2008.

Tabla 2.

Distribución del número de consultas por semestre - años 2006- 2011

\begin{tabular}{cccc}
\hline Año & $\begin{array}{c}\text { Primer } \\
\text { Semestre } \\
\mathbf{n ~ ( \% )}\end{array}$ & $\begin{array}{c}\text { Segundo } \\
\text { Semestre } \\
\mathbf{n}(\%)\end{array}$ & $\begin{array}{c}\text { Total } \\
\mathbf{n}(\%)\end{array}$ \\
\hline 2006 & $154(10.2)$ & $159(10.6)$ & $313(20.8)$ \\
2007 & $128(8.5)$ & $130(8.6)$ & $258(17.1)$ \\
2008 & $129(8.6)$ & $115(7.6)$ & $244(16.2)$ \\
2009 & $111(7.4)$ & $118(7.80)$ & $229(15.2)$ \\
2010 & $89(5.9)$ & $139(8.6)$ & $219(14.5)$ \\
2011 & $94(6.2)$ & $148(9.8)$ & $242(16.2)$ \\
\hline Total & $705(46.8)$ & $800(53.2)$ & $1505(100)$ \\
\hline
\end{tabular}

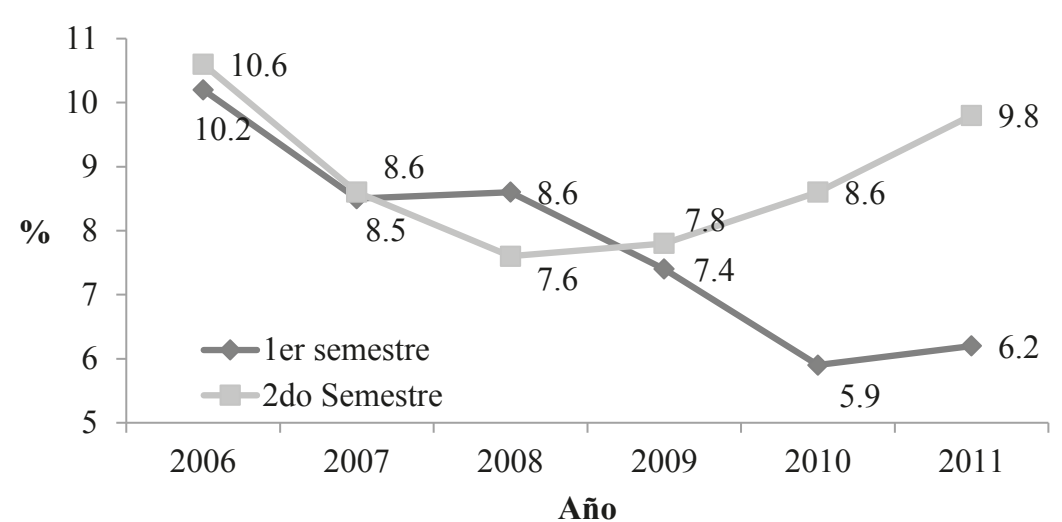

Figura 1.

Distribución porcentual de consultas atenidas en cada año 
Por otro lado, se encontraron un total 2004). La Tabla 3 presenta un resumen de 57 impresiones diagnósticas diferen- de la impresión diagnóstica registrada en tes, las cuales fueron reguladas por los las historias clínicas criterios sustentados en el DSM-IV (APA,

Tabla 3.

Impresión Diagnóstica de los casos atendidos en el CAP

\begin{tabular}{|c|c|c|c|c|c|}
\hline Impresión Diagnóstica & $\mathrm{n}$ & $\%$ & Impresión Diagnóstica & $\mathrm{n}$ & $\%$ \\
\hline Problemas adaptativos & 669 & 14.6 & Trastorno disocial & 6 & .4 \\
\hline Problemas conyugales & 93 & 12.3 & Trastorno explosivo intermitente & 6 & .4 \\
\hline Trastorno depresivo & 88 & 12.2 & Trastorno ciclotímico & 5 & .3 \\
\hline Problemas paterno-filiales & 66 & 10.3 & Fobia específica & 4 & .3 \\
\hline Paciente Sano & 54 & 6.4 & Retraso mental leve & 4 & .3 \\
\hline TDAH & 43 & 4.9 & Trastorno de identidad sexual & 4 & .3 \\
\hline Trastorno de ansiedad & 37 & 4.1 & Enuresis & 3 & .2 \\
\hline Abuso de sustancias & 31 & 3.4 & $\begin{array}{l}\text { Trastorno límite de } \\
\text { la personalidad }\end{array}$ & 3 & .2 \\
\hline Duelo & 22 & 1.8 & Mutismo selectivo & 3 & .2 \\
\hline Problema académico & 20 & 2.9 & T. del control de los impulsos & 3 & .2 \\
\hline Prob. de relación no especif. & 20 & 2.1 & Prob. de relación entre hermanos & 3 & .2 \\
\hline Trastorno del aprendizaje & 20 & 1.7 & Tartamudeo & 2 & .1 \\
\hline T. por estrés postraumático & 19 & 1.4 & Cleptomanía & 2 & .1 \\
\hline Problema biográfico & 17 & 1.3 & Deseo sexual hipoactivo & 2 & .1 \\
\hline Trastorno bipolar & 17 & 1.3 & T. histriónico de la personalidad & 2 & .1 \\
\hline TCA & 13 & 1.4 & T. de ansiedad por separación & 2 & .1 \\
\hline Trastorno depresivo mayor & 13 & 1.4 & T. paranoide de la personalidad & 2 & .1 \\
\hline T. sexual sin especificar & 11 & .8 & Abuso físico del niño & 1 & .1 \\
\hline Crisis de angustia & 11 & .8 & Demencia no especificada & 1 & .1 \\
\hline T. Personalidad no especific. & 10 & .7 & Hipocondría & 1 & .1 \\
\hline T. negativista desafiante & 9 & .6 & Juego patológico & 1 & .1 \\
\hline Fobia social & 8 & .5 & Negligencia de la infancia & 1 & .1 \\
\hline T. personalidad por dependenc. & 8 & .5 & Trastorno de Asperger & 1 & .1 \\
\hline T. personalidad por evitación & 8 & .5 & Tricotilomanía & 1 & .1 \\
\hline Abuso sexual & 6 & .4 & Sin dato & 111 & 7.4 \\
\hline Trastorno del sueño & 6 & .4 & & & \\
\hline
\end{tabular}

Nota: $T=$ Trastorno, Prob. $=$ Problemas 
Tabla 4.

Comparación de impresiones diagnósticas entre varones y mujeres

\begin{tabular}{|c|c|c|c|c|}
\hline \multirow{2}{*}{$\begin{array}{l}\text { Impresiones } \\
\text { Diagnósticas }\end{array}$} & \multicolumn{2}{|c|}{ Varones } & \multicolumn{2}{|c|}{ Mujeres } \\
\hline & $\mathrm{N}$ & $\%$ & $\mathrm{n}$ & $\%$ \\
\hline Prob. adaptativo & 318 & 41.4 & 351 & 47.7 \\
\hline Prob. conyugales & 57 & 7.4 & 36 & 4.9 \\
\hline Trastorno depresivo & 47 & 6.1 & 41 & 5.6 \\
\hline Prob. paterno-filiales & 46 & 6 & 20 & 2.7 \\
\hline Paciente Sano & 33 & 4.3 & 21 & 2.9 \\
\hline TDAH & 11 & 1.4 & 32 & 4.3 \\
\hline T. de ansiedad & 19 & 2.5 & 18 & 2.4 \\
\hline Abuso de sustancias & 15 & 2 & 16 & 2.2 \\
\hline Prob. académico & 7 & .9 & 13 & 1.8 \\
\hline Prob. relación NE & 11 & 1.4 & 9 & 1.2 \\
\hline Trastorno del Apzje. & 8 & 1.0 & 12 & 1.6 \\
\hline Duelo & 11 & 1.5 & 11 & 1.5 \\
\hline Problema biográfico & 9 & 1.2 & 8 & 1.1 \\
\hline T. conducta alimentar. & 7 & .9 & 6 & .8 \\
\hline T. depresivo mayor & 8 & 1.0 & 5 & .7 \\
\hline T. obsesivo-compulsiv & 7 & .9 & 5 & .7 \\
\hline T. estrés postrauma & 11 & 1.4 & 8 & 1.0 \\
\hline T. bipolar & 9 & 1.2 & 2 & .3 \\
\hline Crisis de angustia & 4 & .5 & 7 & 1 \\
\hline T. personalidad NE. & 7 & .9 & 3 & .4 \\
\hline Trastorno sexual NE & 4 & .5 & 5 & .7 \\
\hline T. negativista desaf. & 4 & .5 & 5 & .7 \\
\hline Fobia social & 6 & .8 & 2 & 0,3 \\
\hline T. personal. dependen. & 6 & .8 & 2 & 0,3 \\
\hline T. personal. evitación & 4 & .5 & 4 & 0,5 \\
\hline Trastorno disocial & 1 & .1 & 5 & 0,7 \\
\hline
\end{tabular}

\begin{tabular}{|c|c|c|c|c|}
\hline \multirow{2}{*}{$\begin{array}{l}\text { Impresiones } \\
\text { Diagnósticas }\end{array}$} & \multicolumn{2}{|c|}{ Varones } & \multicolumn{2}{|c|}{ Mujeres } \\
\hline & $n$ & $\%$ & $\mathrm{n}$ & $\%$ \\
\hline Trastorno distímico & 5 & .7 & 1 & .1 \\
\hline T. explosivo intermit. & 6 & .8 & 0 & 0 \\
\hline Abuso sex. & 4 & .5 & 2 & .3 \\
\hline Trastorno del sueño & 4 & .5 & 2 & .3 \\
\hline Fobia específica & 3 & .4 & 1 & .1 \\
\hline T. de identidad sex. & 2 & .3 & 2 & .3 \\
\hline Retraso mental leve & 1 & .1 & 3 & .4 \\
\hline T. control impulsos & 1 & .1 & 2 & .3 \\
\hline P. relación Hnos. & 3 & .4 & 0 & 0 \\
\hline Mutismo selectivo & 1 & .1 & 2 & .3 \\
\hline T. límite personalid. & 0 & 0 & 3 & .4 \\
\hline Enuresis & 1 & .1 & 2 & .3 \\
\hline T. histriónico & 1 & .1 & 1 & .1 \\
\hline T. de ansiedad & 0 & 0 & 2 & .3 \\
\hline Tartamudeo & 1 & .1 & 1 & .1 \\
\hline T. paranoide & 2 & .3 & 0 & 0 \\
\hline Deseo sex.hipoactivo & 2 & .3 & 0 & 0 \\
\hline Cleptomanía & 0 & 0 & 2 & .3 \\
\hline Negligencia del inf. & 1 & .1 & 0 & 0 \\
\hline T. orgásmico fem. & 0 & 0 & 1 & .1 \\
\hline Demencia NE & 1 & .1 & 0 & 0 \\
\hline Tricotilomanía & 1 & .1 & 0 & 0 \\
\hline Hipocondría & 0 & 0 & 1 & .1 \\
\hline Juego patológico & 0 & 0 & 1 & .1 \\
\hline T. de Asperger & 0 & 0 & 1 & .1 \\
\hline Abuso físico del inf. & 0 & 0 & 1 & .1 \\
\hline
\end{tabular}

$\mathrm{T}=$ Trastorno, Prob=Problema, NE $=$ No especificado

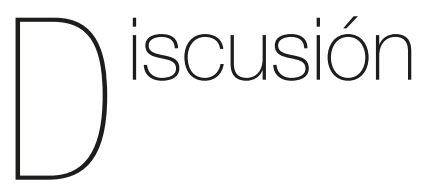

la comparación de las impresiones diagnósticas entre género permite hacer algunos comentarios al respecto. Inicialmente es importante destacar que la distribución entre ambos géneros (varones-mujeres)

Los objetivos relacionados con identipresenta un porcentaje relativamente hoficar la evolución del número de consultas en los años comprendidos en el estudio y mogéneo al momento de identificar las personas que emplean los servicios del 
CAP, observándose mas consultas en los varones (51.1\%) en comparación con las mujeres (48.9\%). En otros trabajos (Muñoz-Martínez \& Novoa-Gómez, 2012) con pretensiones similares a la presente investigación se observaron similares comportamientos en cuanto a la distribución por género. Si bien esta situación no corresponde a la tendencia, lo observado en varios reportes (Ballesteros et al., 2006; Kother et al., 2011; Londoño et al., 2010; Moreno et al., 2012; Suriá-Martínez, Ordóñez, \& Martínez-Maciá, 2011; Quesada, 2004) señalan una mayor tendencia de las mujeres en la utilización del servicio de atención psicológica. La importancia de tener en cuenta el género en estudios relacionados con los procesos de salud mental, permitirá dilucidar factores de predisposición que son asociados a las funciones de roles sociales.

En el primer objetivo estudiado, se encontró que la evolución de consultas por año presenta comportamientos irregulares, tanto en el análisis de cada año como la comparación por semestres. No hay investigaciones que registren una cifra de igualdad en la cantidad de consultas en los periodos analizados. En concordancia con lo expresado anteriormente, Loubat y Magaña (2005) analizaron las consultas entre los años 2001 y 2005 en los diferentes focos o unidades atencionales del CAP de la Universidad de Santiago de Chile, y hallaron un patrón irregular en este periodo, observando disminución de usuarios adultos y aumento de consultas en poblaciones infanto-adolescentes. Por otro lado, otros estudios en contextos con población de universitarios españoles, han observado un incremento de los motivos de consulta (Ávila, Herrero, \& Fernández, 2009; Saúl et al., 2009; Suriá-Martínez et al., 2011). Los posibles descensos observados entre los años 2007 y 2010 pueden entenderse debido a situaciones particulares del CAP de la Universidad de Manizales donde se evidenciaron cambios estructurales como por el ejemplo el cambio de sede.

Este suceso tiene sentido si se logra entender que la amplia demanda de consultas de estos centros está primordialmente concentrada por la población estudiantil universitaria, (Quesada, 2004), además que una porción considerable de la misma es adolescente, la cual se debe enfrentar a los cambios de adaptación exigidos por contextos nuevos y difíciles como es el ambiente de la educación superior. En consecuencia, cuando se reconoce que los motivos de consulta son en esencia fenómenos dados por las condiciones sociales (Loubaty Magaña, 2005; Quesada, 2004) y personales (MuñozMartínez \& Novoa-Gómez 2012), al igual, como la multifactoriedad en la deserción de los procesos terapéuticos (BuelaCasal, Sierra, López, \& Rodríguez, 2001; Rondón, Otálora, \& Salamanca 2009), se subrayan con énfasis las variadas características dadas en escenarios como éste y permite hacer una lectura amplia de los aspectos ocurridos en la adherencia o deserción, así como de aumento o disminución de las frecuencias de consultantes acompañados en los procesos terapéuticos, y principalmente si se tiene en cuenta la población infanto-adolescente como periodos del ciclo vital de elevada sensibilidad.

Frente al segundo objetivo, las impresiones diagnósticas evidenciaron datos que describen que los problemas de adaptación son los más frecuentes, seguidos de los problemas conyugales y trastornos de depresión. En cuanto a la 
comparación entre géneros, las mujeres presentan mayores porcentajes en problemas de adaptación, mientras que en los varones prevalecen los problemas conyugales, depresivos y paterno-filiales. Así, los presentes resultados tienen parcialmente coherencia con la literatura internacional y nacional. Las referencias en la literatura muestran que los primeros renglones de las prevalencias están identificados con los trastornos de ánimo (depresión), lo cual señala como tendencia de los principales resultados estudios de salud mental a nivel de Latinoamérica (Rodríguez, Khony, \& Aguilar-Gaxiola, 2009). Además que están acompañados de una variedad de problemas o entidades nosológicas que varían según el género, la edad y el contexto sociocultural.

En el Estudio Nacional de Salud Mental, realizado en Colombia en el año 2003 (Posada Villa, Aguilar Gaxiola, Magaña, \& Gómez, 2004), se encontró que las fobias específicas fueron las más comunes seguidas por el trastorno depresivo mayor, el trastorno de ansiedad por separación y el abuso de alcohol. De otra parte, en la población clínica infantil y adolescente española, la mayor prevalencia de categorías diagnósticas observadas correspondió a los trastornos de conducta, ansiedad, depresión, del desarrollo, y de eliminación (Aláez Fernández, MartínezArias, \& Rodríguez-Sutil, 2000). Aunque, según afirma De la Barra (2009), las prevalencias de trastornos mentales varía entre 1 - 51\% en poblaciones de niños y adolescentes, acorde a la revisión de 52 estudios epidemiológicos, este amplio rango se debe a los criterios e instrumentos utilizados; otras cifras sugieren que a nivel mundial la prevalencia de trastornos psiquiátricos corresponde entre el 8\% y 45\% (Costello, Egger, \& Angold, 2005).
En otro estudio, los diagnósticos con mayor proporción de gravedad fueron los trastornos de ánimo, observados en una muestra de adolescentes mexicanos (Benjet et al., 2009); asimismo, hallaron que las mujeres adolescentes presentan mayor prevalencia en cualquier trastorno en comparación a los hombres.

De esta forma, en contextos escolares, principalmente en los últimos años a nivel de secundaria y el periodo de transición a la educación superior, son edades (16 a 22 años) de mayor consulta en el CAP (34.1\%), se establecen datos que van de la línea de los presentes resultados. Así, por ejemplo, Moreno et al. (2011) encontraron en una población universitaria que los problemas más frecuentes fueron problemas de conducta (que incluyen los trastornos por déficit de atención con hiperactividad, negativista desafiante y disocial) depresión, personalidad, paterno filiales, ansiedad, aprendizaje, problemas en la relación de pareja, retardo mental y otros; resultados que se asemejan a los hallados en el presente trabajo. Otro antecedente en universidades españolas, también estableció que los problemas más atendidos en estos servicios son ansiedad, estado de ánimo, desadaptación, déficit de autoestima o habilidades sociales, ansiedad ante los exámenes, problemas de relación interpersonal, entre otros (Saúl et al., 2009).

Todo lo anterior permite establecer algunas implicaciones que se dan en el siguiente orden: primero, se reconoce que la infancia es importante para identificar algunos de los problemas frecuentes en este rango de edad; pero, más aún, reviste particular interés lo observado en la adolescencia, ya que corresponde a un periodo de múltiples cambios y 
asimilaciones de distinto orden (biológico, personal, social, etc.), y es quizás donde mejor se logran identificar algunas diferencias entre géneros, ante lo cual se han establecido estudios que afirman que la adolescencia parece ser una edad que es más vulnerable para las mujeres que para los hombres (Benjet et al., 2009). Esto puede entenderse por dos aspectos que la literatura está problematizando y las investigaciones se ubican en esta dirección, y estos radican en teorías biológicas y socioculturales (Arenas \& Puigcerver, 2009), como factores de predisposición que aproximan explicaciones del predominio femenino en la prevalencia de problemas mentales.

Un segundo aspecto, ligado al comentario anterior, corresponde a la edad de inicio de los trastornos. Algunos autores consideran que la edad de inicio usual de los trastornos mentales es la infancia y la adolescencia (Kessler et al., 2005). Otros informes precisan que los trastornos de conducta alimentaria tienen curvas de inicio en la adolescencia (Swanson, Crow, Le Grange, Swendsen, \& Merikangas, 2011), de modo que es en las edades tempranas donde se observan entidades nosológicas como la ansiedad (fobias específicas, fobias por separación y TDAH) correspondiente al rango de edad entre los 5 y años; en tanto la ansiedad generalizada, los trastornos de ánimo e impulsividad, ocurren entre los 11 y 12 años. Después, entre los 13 y 14 años aparecen los trastornos por uso de sustancias (Benjet et al., 2009).

Finalmente, una tercera implicación se sintetiza en la dirección oportuna de programas de promoción de la salud mental y prevención de la enfermedad y, desde luego, el tratamiento a tiempo. Aunque, a diferencia de otros continentes, para América Latina la brecha es demasiado amplia entre quienes reciben atención y los que no (Kohn et al., 2005). Adicionalmente, Kohn y Rodríguez (2009) afirman que también puede entenderse que a los aspectos relacionados con la salud mental no se les ha dado la debida importancia, producto de diversos factores sociales y gubernamentales. En los países industrializados se da un cambio en la importancia del papel de la atención de la salud mental, situación que para los países en vía de desarrollo pasa desapercibida y sin la relevancia que debería dársele. A pesar de ello, el panorama puede parecer alentador cuando se considera que quienes asisten a estos centros de atención psicológica corresponden a jóvenes que cursan alguno nivel de escolaridad, lo cual permitirá que tanto en los procesos académicos como personales y sociales estén acompañados por programas y recursos de afrontamiento, en los ya mencionados periodos críticos como la adolescencia. Como lo expresan Kohn et al. (2005) es preciso reconocer que las cifras de prevalencia no son únicamente datos de estados psicopatológicos; son la resultante de un conjunto de características y problemas adyacentes, en los cuales el comportamiento tiene un rol sobresaliente (Campo-Arias, \& Cassiani, 2008; Kohn et al., 2005).

De modo que la propuesta de una red de CAP y USP entrelazados con líneas de investigación permitiría que se establezcan directrices sobre toma de decisiones de acuerdo con los resultados de estudios epidemiológicos asociados a salud mental. Es necesario advertir algunas de las limitaciones que se presentaron en el trabajo, entre las cuales se destaca principalmente alguna información no 
diligenciada por los estudiantes practicantes. Estos datos pueden orientar con mayor precisión sobre las características y variables tratadas; esta observación también es recogida en un trabajo previo realizado por López, Montoya y Dussán (2012). Otro elemento, corresponde a los criterios con que se emiten las impresiones diagnósticas, las cuales en ocasiones pueden ser difusas y poco claras.

Por último, estos hallazgos permiten a la comunidad académica y profesional enfatizar en la importancia e impacto que tienen los Programas de Psicología de las universidades del país con la extensión de los CAP y USP encadenados a procesos de investigación que faciliten tanto a la población escolar como a la comunidad en general contar con tales servicios.

\section{Agradecimientos}

Los autores quieren extender su agradecimiento a estudiantes practicantes y profesores asesores que hacen parte del Centro de Atención Psicológica de la Universidad de Manizales.

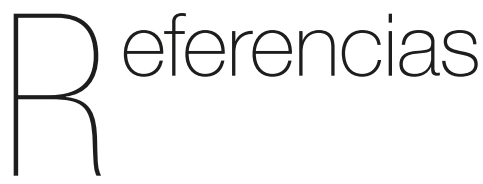

Aláez Fernández, M., Martínez-Arias, R., \& Rodríguez-Sutil, C. (2000). Prevalencia de trastornos psicológicos en niños y adolescentes, su relación con la edad y el género. Psicothema, 12(4), 525-532.
Arenas, M., \& Puigcerver, A. (2009). Diferencias entre hombres y mujeres en los trastornos de ansiedad: una aproximación psicobiológica. Escritos de Psicología, 3(1), 20-29.

Asociación Americana de Psiquiatría. (2004). DSM-IV-R. Manual diagnóstico y estadístico de los trastornos mentales. Barcelona: Masson.

Ávila Espada, A., Herrero Sánchez, J., \& Fernández Martín L.C. (2009). Más allá de la docencia: la unidad de atención psicológica y salud mental del universitario (UAPSMU) en la universidad de Salamanca (1996-2004). Acción Psicológica, 6, 49-62.

Ballesteros, B. P., Medina, A., \& Caycedo, C. (2006). El bienestar psicológico definido por asistentes a un servicio de consulta psicológica en Bogotá, Colombia. Universitas Psycholgica, 5(2), 239-258.

Bausela, E. (2005). Diseño de un servicio de orientación psicológica en el contexto de la Universidad de León. Revista de Psicodidáctica, 10(2), 123-139.

Benjet, C., Borges, G., Medina-Mora, M. E., Méndez, E., Fleiz, C., Rojas, E., \& Cruz, C. (2009). Diferencias de sexo en la prevalencia y severidad de trastornos psiquiátricos en adolescentes de la Ciudad de México. Salud mental, 32(2), 155-163.

Benjet, C., Borges, G., Medina-Mora, M. E., Blanco, J., Rojas, E., Fleiz, C., \& AguilarGaxiola, S. (2009). La encuesta de salud mental en adolescentes de México. En: J. Rodriguez, R. Khony S. Aguilar-Gaxiola (Eds.), Epidemiología de los trastornos mentales en América Latina y el Caribe. (pp.90-98). Washington, D.C.: OPS. 
Bisquerra, R. (1998). Modelos de orientación e intervención psicopedagógica. Barcelona: Praxis

Buela-Casal, G., Sierra, J., López, M., \& Rodríguez, I. (2001). Habilidades terapéuticas y del terapeuta. En: G. Buela-Casal, \& J. Sierra (Eds.). Manual de Evaluación y Tratamientos Psicológicos. (pp. 25-39). Madrid, España: Biblioteca Nueva.

Calvopiña, G., \& Rojeab, B. (2011). Las psicoterapias en los Centros de Atención Psicológica de las universidades PUCE, UCE, UDLA Y UPA del Distrito Metropolitano de Quito, período 2010 2011. Facultad de Psicología. PUCE. Quito. Recuperado de http://repositorio.puce. edu.ec/handle/22000/3451

Campo-Arias, A., \& Cassiani, C. (2008). Trastornos mentales más frecuentes: prevalencia y algunos factores sociodemográficos asociados. Revista Colombiana de Psiquiatría, 37(4), 598-613.

Castonguay, L., Locke, B., \& Hayes, J. (2011). The Center for Collegiate Mental Health: AnExample of a Practice-Research Network in University Counseling Centers. Journal of College Student Psychotherapy, 25(2), 105-119.

Colegio Colombiano de Psicólogos. (2012). Deontología y bioética del ejercicio de la psicología. Bogotá: Colegio Colombiano de Psicólogos.

Costello, E. J., Egger, H., \& Angold, A. (2005). 10-year re- search update review: The epidemiology of child and adolescent psychiatric disorders: I. Methods and public health burden. Journal of the American
Academy of Child and Adolescent Psychiatry, 44, 972-986.

De la Barra M, F. (2009). Epidemiología de trastornos psiquiátricos en niños y adolescentes: Estudios de prevalencia. Revista Chilena de Neuropsiquiatría, 47(4), 303-314.

Hayes, J., Locke, B., \& Castonguay, L. (2011). The Center for Collegiate Mental Health: Practice and Research Working Together. Journal of College Counseling, 14(2), 101-104.

Kessler, R. C., Berglund, P., Demler, O., Jin, R., Merikangas, K. R., \& Walters, E. E. (2005). Lifetime prevalence and age-of-onset distributions of DSM-IV disorders in the National Comorbidity Survey Replication. Archives of general psychiatry, 62(6), 593.

Kohn, R., \& Rodríguez, J. (2009). Prevalencia y carga de los trastornos mentales en la población adulta de América Latina y el Caribe. En: J. Rodriguez, R. Khony S. Aguilar-Gaxiola (Eds.). Epidemiología de los trastornos mentales en América Latina y el Caribe. (pp. 3-17). Washington, D.C. OPS.

Kohn, R., Levav, I., Caldas de Almeida, J. M., Vicente, B., Andrade, L., Caraveo-Anduaga, J. J., Saxena, S., \& Saraceno, B. (2005). Los trastornos mentales en América Latina y el Caribe: asunto prioritario para la salud pública. Revista Panamericana de Salud Publica, 18(4/5), 229-40.

Kother, M., Aguilar, M., Lisboa, R., Freitas, R., Machado, S., \& Gomes, T. (2011). Motivos de busca de atendimento psicológico por adolescentes em uma clínica-escola. Psicologia: Teoria e Prática, 13(2), 63-756. 
Londoño, H. N., Marín C., Juárez, F., Palacio, J., Muñiz, O., Escobar, B. Herron, I., \& López, I. C. (2010). Factores de riesgo psicosociales y ambientales asociados a trastornos mentales. Suma Psicológica, 17(1), 59-68.

López, M. R., Montoya, D. M., \& Dussán, C. (2012). Caracterización de los asistentes al Centro de Atención Psicológica de la Universidad de Manizales, 2006-2010. Hacia la Promoción de la Salud, 17(2), 149-166.

Loubat, M., \& Magaña, I. (2005). Centro de Atención Psicológica de la Escuela de Psicología de la Universidad de Santiago de Chile: una experiencia de reestructuración inspirada en los modelos biopsicosocial y de salud pública. Revista de Psicología de la Universidad de Chile, 14(2), 16.

Montoya, D. P. (2011). Caracterización de los programas de formación en psicología en Colombia. Bogotá: Asociación Colombiana de Facultades de Psicología.

Montero, I., \& León, O. G. (2005). Sistema de clasificación del método en los informes de investigación en Psicología. International Journal of Clinical and Health Psychology, 5, 115-127.

Montero, I., \& León, O. (2007). A guide for naming research studies in Psychology. International Journal of Clinical and Health Psychology, 7(3), 847-862.

Montero, I., \& León, O. G. (2005). Sistema de clasificación del método en los informes de investigación en Psicología. International Journal of Clinical and Health Psychology, 5, 115-127.
Moreno, L. H., Rozo, M. M., Cantor, M. I., \& Toro, R. (2012).Características psicosociales de la población asistente a la unidad de servicios psicológicos de la universidad católica de Colombia durante los años 2007 y 2008. Psychologia: avances de la disciplina, 6(1), 21-33.

Muñoz-Martínez, A. M., \& Novoa-Gómez. M. (2012). Motivos de consulta e hipótesis clínicas explicativas. Terapia psicológica, 30(1), 25-36.

Organista, P., \& Garzón, A. (2012). Panorámica actual sobre la formación de los psicólogos en Colombia. En: Colegio Colombiano de Psicólogos (Eds.). Condiciones sociodemográficas educativas laborales y salariales del psicólogo Colombiano. (pp. 67-86). Recuperado de http://www. colpsic.org.co/documentos/condiciones_ del_psicologo.pdf.

Posada Villa, J. A., Aguilar Gaxiola, S., Magaña, C. G., \& Gómez, L. C. (2004). Prevalencia de trastornos mentales y uso de servicios: resultados preliminares del Estudio Nacional de Salud Mental, Colombia, 2003. Revista Colombiana de Psiquiatría, 33(3), 241-62.

Quesada, S. (2004). Estudio sobre los motivos de consulta psicológica en una población universitaria. Universitas Psychologica, 3, 7-16.

Red de Instituciones de Servicios Universitarios de Atención Psicológica. (2007). Caracterización de la población que consulta a las ISUAP y sus motivos de consulta. Recuperado de: www.ascofapsi. org.co/documentos/.../presentacion_ encuentro.ppt. 
Rodríguez, J., Khony, R., \& Aguilar-Gaxiola, S. (2009). Epidemiología de los trastornos mentales en América Latina y el Caribe. Washington, D.C. OPS.

Rondón, A. P., Otálora, I. L., \& Salamanca, Y. (2009). Factores que influyen en la deserción terapéutica de los consultantes de un centro universitario de atención psicológica. International Journal of Psychological Research, 2(2), 137-147.

Saúl, L.A., López G., M.A., \& Bermejo, B.G. (2009). Los Servicios de Atención Psicológica en las Universidades Españolas. Acción Psicológica, 6, 17-40.

Saxena, S., Thornicroft, G., Knapp, M., \& Whiteford, H. (2007). Global Mental Health 2-Resources for mental health: scarcity, inequity, and inefficiency. Lancet, 370(9590), 878-889.

Sierra, Y., \& Fonseca, M. A. (2012). Situación laboral y ocupacionalde los psicólogos colombianos. En: Colegio Colombiano de Psicólogos (Eds.). Condiciones sociodemográficas educativas laborales y salariales del psicólogo Colombiano. (pp.87-114). Recuperado de http://www. colpsic.org.co/documentos/condiciones_ del_psicologo.pdf
Suriá-Martínez, R., Ordóñez, T., \& MartínezMaciá, D. (2011). Estudio descriptivo de la evolución del servicio de asesoramiento psicológico y psicoeducativo de la Universidad de Alicante. REOP, 22(2), 151-164.

Swanson, S. A., Crow, S. J., Le Grange, D., Swendsen, J., \& Merikangas, K. R. (2011). Prevalence and correlates of eating disorders in adolescents: results from the national comorbidity survey replication adolescent supplement. Archives of General Psychiatry, 68(7), 714.

Viñas, B. M., \& Hernández, D. A. (2012). Estrategias para fortalecer la competencia del psicólogo en la práctica psicoterapéutica. Enseñanza e investigación en Psicología 17(1), 101-116. 